\title{
Sensitivity of the electrocardiography inverse solution to the torso conductivity uncertainties
}

\author{
N. ZemZemi ${ }^{1}$, R. Aboulaich ${ }^{2}$, N. FIKAL ${ }^{2}$, E. El GUARMAH ${ }^{3}$ \\ 1 Inria Bordeaux Sud-Ouest Carmen Project, France \\ ${ }^{2}$ LERMA Mohammadia Engineering School, Morocco \\ 3 BEFRA, Royal Air Force school, Morocco
}

\begin{abstract}
Electrocardiography imaging (ECGI) is a new non invasive technology used for heart diagnosis. It allows to construct the electrical potential on the heart surface only from measurement on the body surface and some geometrical informations of the torso. The purpose of this work is twofold: First, we propose a new formulation to calculate the distribution of the electric potential on the heart, from measurements on the torso surface. Second, we study the influence of the errors and uncertainties on the conductivity parameters, on the ECGI solution. We use an optimal control formulation for the mathematical formulation of the problem with a stochastic diffusion equation as a constraint. The descretization is done using stochastic Galerkin method allowing to separate random and deterministic variables. The optimal control problem is solved using a conjugate gradient method where the gradient of the cost function is computed with an adjoint technique. The efficiency of this approach to solve the inverse problem and the usability to quantify the effect of conductivity uncertainties in the torso are demonstrated through a number of numerical simulations on a $2 \mathrm{D}$ geometrical model. Our results show that adding $\pm 50 \%$ uncertainties in the fat conductivity does not alter the inverse solution, whereas adding $\pm 50 \%$ uncertainties in the lung conductivity affects the reconstructed heart potential by almost $50 \%$.
\end{abstract}

\section{Introduction}

The ECGI procedure helps medical doctors to target some triggers of cardiac arrhythmia and consequently plan a much more accurate surgical interventions [1]. The mathematical problem behind the ECGI solution is known to be ill posed [2]. Therefore, many regularization techniques have been used in order to solve the problem [3-7]. Thus, the obtained solution depends on the regularization method and parameters [8]. Furthermore, the inverse solution does not depend only on the regularization method but also to the physical parameters and the geometry of the patient. These dependencies have not been taken into account in most (if not all) of the studies. In particular, in most of the studies in the literature the torso is supposed to be a homogenous. Moreover, when the conductive heterogeneities are considered, they are fixed according to data obtained from textbooks. The problem is that these data are different from a paper to another [9-11]. Their variability is mainly due to the difference between the experiment environments and other factors related to the measurement tools. Only few works have been performed in order to study the effect of conductivities uncertainty in the propagation 
of the electrical potential in the torso [12-14]. In [13], authors use the stochastic finite elements method (SFEM) to evaluate the effect of lungs muscles and fat conductivities on the forward problem. In [14], authors used a principal component approach to predict the effect of conductivities variation on the body surface potential.

However, to the best of our knowledge, no work in the literature has treated the influence of conductivity uncertainties of the ECGI inverse solution. In this work, we propose to use an optimal control approach to solve the inverse problem and to compute the potential value on the heart, we use an energy functional [15]. with SPDE constraints as proposed in [13]. We are interested in assessing the effect of the variability of tissue conductivity on the solution of the ECGI problem. The resolution of optimal control will be made using an iterative procedure based on the conjugate gradient method and the numerical approximation will be performed using the SFEM.

\section{Methods}

\subsection{Solving stochastic forward problem of electrocardiography}

Following [13], we represent the stochastic characteristics of the forward solution of the Laplace equation by the generalized chaos polynomial. For the space domain we use simplified analytical 2D model representing a cross-section of the torso (see Figure 1) in which the conductivities vary stochastically. Let us first denote by $D$ the spatial domain and $\Omega$ the probability space. By supposing that the conductivity parameter depends on the space and on the stochastic variable $\sigma(x, \xi)$, the solution of the Laplace equation does also depend on space and the stochastic variable $u(x, \xi))$. The stochastic forward problem of electrocardiography can be written as follows:

$$
\left\{\begin{array}{l}
\nabla \cdot(\sigma(x, \xi) \nabla u(x, \xi))=0 \quad \text { in } D \times \Omega \\
u(x, \xi)=u_{0} \text { on } \Gamma_{\mathrm{int}} \times \Omega \\
\sigma(x, \xi) \frac{\partial u(x, \xi)}{\partial n}=0 \quad \text { on } \Gamma_{\mathrm{ext}} \times \Omega
\end{array}\right.
$$

where, $\Gamma_{\text {int }}$ and $\Gamma_{\text {ext }}$ are the epicardial and torso boundaries respectively, $\xi \in \Omega$ is the stochastic variable (it could also be a vector) and $u_{0}$ is the potential at the epicardial boundary.

\subsection{Numerical descretization of the stochastic forward problem}

We use the stochastic Galerkin (SG) method in order to solve equation (1). A stochastic process $X(\xi)$ of a parameter or a variable $X$ is represented by weighted sum of orthogonal polynomials known as probability density functions $\operatorname{PDFs}\left\{\Psi_{i}(\xi)\right\}$. More details about the different choices of PDFs could be found in [16]. In our case we use the Legendre polynomials which are more suitable for uniform probability density .

$$
X(\xi)=\sum_{i=0}^{p} \hat{X}_{i} \Psi_{i}(\xi)
$$

where $\hat{X}_{i}$ are the projections of the random process on the stochastic basis $\left\{\Psi_{i}(\xi)\right\}_{i=1}^{p}$. The mean value and the standard deviation of $\mathrm{X}$ over $\Omega$ are then computed as follows

$$
\mathrm{E}(X)=\int_{\Omega} \sum_{i=0}^{p} \hat{X}_{i} \Psi_{i}(\xi)=\hat{X}_{0}, \quad \operatorname{stdev}[X]=\left(\sum_{i=1}^{p} \hat{X}_{i}^{2} \int_{\Omega} \Psi_{i}(\xi)^{2}\right)^{\frac{1}{2}}
$$


Since in our study we would like to evaluate the effect of the conductivity randomness of the different torso organs on the electrical potential. Both of $\sigma$ and $u$ are now expressed in the Galerkin space as follows:

$$
\sigma(x, \xi)=\sum_{i=0}^{p} \hat{\sigma}_{i}(x) \Psi_{i}(\xi), \quad u(x, \xi)=\sum_{j=0}^{p} \hat{u}_{j}(x) \Psi_{i}(\xi)
$$

By substituting (2),(3) into the elliptic equation (1) and by projecting the result on the polynomial basis $\left\{\Psi_{k}(\xi)\right\}_{k=1}^{p}$ we obtain the following system: For $k=0, \ldots, p$ :

$$
\begin{aligned}
\left.\sum_{i=0}^{p} \sum_{j=0}^{p} T_{i j k} \nabla \cdot\left(\hat{\sigma}_{i}(x) \nabla\right) \hat{u}_{j}(x)\right) & =0 \text { in } D, \\
\hat{u}_{0}(x) & =u_{0}(x) \text { on } \Gamma_{\mathrm{int}}, \\
\hat{u}_{j}(x) & =0 \text { on } \Gamma_{\mathrm{int}} \quad \forall j=1, \ldots p, \\
\hat{\sigma}_{i}(x) \frac{\partial \hat{u}_{j}(x)}{\partial n} & =0 \text { on } \Gamma_{\mathrm{ext}} \forall i, j=0, \ldots p,
\end{aligned}
$$

where $T_{i j k}=E\left[\Psi_{i}(\xi), \Psi_{j}(\xi), \Psi_{k}(\xi)\right]$. By applying the standard finite elements variational formlation and Galerkin projections we obtain a linear system of size $(p \times d o f)$, where dof is the number of the degrees of freedom for the Laplace equation in the deterministic framework.

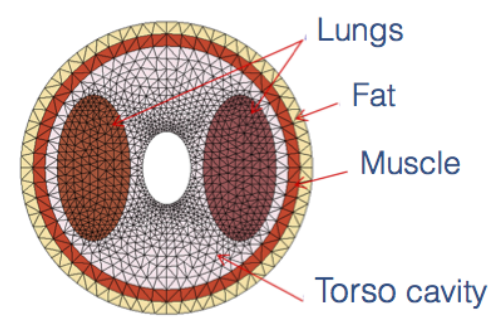

Fig. 1: 2D computational mesh of the torso geometry showing the different regions of the torso considered in this study (fat, muscle, lungs, torso cavity).

\subsection{Stochastic inverse problem problem}

In this section we propose to build an optimal control problem taking into account the variability of the tissue conductivities in the torso. Let us first propose a gold standard solution representing the true electrical potential in the torso. Supposing that the true conductivity distribution in the torso is $\sigma_{T}$, at a given time the true electrical potential in the torso $u_{T}$ is governed by the Laplace equation, it depends on the extracellular potential at the epicardium $u_{e x}$ as follows:

$$
\left\{\begin{array}{l}
\nabla \cdot\left(\sigma_{T} \nabla u_{T}\right)=0 \quad \text { in } D \\
u_{T}=u_{e x} \text { on } \Gamma_{\mathrm{int}}, \\
\sigma_{T} \frac{\partial u_{T}}{\partial n}=0 \text { on } \Gamma_{\mathrm{ext}} .
\end{array}\right.
$$


From the deterministic forward problem solution $u_{T}$, we extract the electrical potential at the external boundary and we denote it by $f=u_{T} / \Gamma_{\mathrm{ext}}$. For the inverse problem formulation, we write a cost function that takes into account the uncertainties in the torso conductivities. We then use an energy cost function as used in $[17,15]$ constrained by the stochastic conductivity Laplace formulation as presented in the previous paragraph. We look for the current density and the value of the potential on the epicardial boundary $(\eta, \tau) \in L^{-\frac{1}{2}}\left(\Gamma_{\text {int }}\right) \times L^{\frac{1}{2}}\left(\Gamma_{\text {int }}\right)$ minimizing the following cost function

$$
\left\{\begin{array}{l}
J(\eta, \tau)=\frac{1}{2} E\left(\|v(x, \xi)-f\|_{L^{2}\left(\Gamma_{\mathrm{ext}}\right)}^{2}+\frac{1}{2}\left\|\sigma(x, \xi) \frac{\partial u(x, \xi)}{\partial n}-\eta\right\|_{L^{2}\left(\Gamma_{\mathrm{int}}\right)}^{2}\right) \\
\text { with } v(x, \xi) \text { solution of : } \\
\nabla \cdot(\sigma(x, \xi) \nabla v(x, \xi))=0 \text { in } D \times \Omega, \\
v(x, \xi)=\tau \text { on } \Gamma_{\mathrm{int}} \times \Omega, \\
\sigma(x, \xi) \frac{\partial v(x, \xi)}{\partial n}=0 \quad \text { on } \Gamma_{\mathrm{ext}} \times \Omega .
\end{array}\right.
$$

In order to solve this minimization problem, we use a conjugate gradient method as used in [15] where the components of the gradient of the cost function are computed using an adjoint method. The gradient of the functional $J$ is given by:

$$
\left\{\begin{array}{l}
<\frac{\partial J(\eta, \tau)}{\partial \eta} \cdot \phi>=-E\left[\int_{\Gamma_{\mathrm{int}}}\left(\sigma \frac{\partial v}{\partial n}-\eta\right) \phi d \Gamma_{\mathrm{int}}\right] \quad \forall \phi \in L^{2}\left(\Gamma_{\mathrm{int}}\right), \\
<\frac{\partial J(\eta, \tau)}{\partial \tau} \cdot h>=E\left[\int_{\Gamma_{\mathrm{int}}} \sigma \frac{\partial \lambda}{\partial n} h d \Gamma_{\mathrm{int}}\right] \quad \forall h \in L^{2}\left(\Gamma_{\mathrm{int}}\right), \\
\text { with } \lambda \text { solution of : } \\
\nabla \cdot(\sigma(x, \xi) \nabla \lambda(x, \xi))=0 \quad \text { on } D \times \Omega \\
\lambda(x, \xi)=\sigma(x, \xi) \frac{\partial v(x, \xi)}{\partial n}-\eta \quad \text { on } \Gamma_{\mathrm{int}} \times \Omega, \\
\sigma(x, \xi) \frac{\partial \lambda(x, \xi)}{\partial n}=-(v-f) \quad \text { on } \Gamma_{\mathrm{ext}} \times \Omega .
\end{array}\right.
$$

\subsection{The conjugate gradient algorithm.}

The stochastic Cauchy problem has been reformulated as a minimization one in the previous section. For the numerical solution, we use a conjugate gradient optimization procedure. The algorithm is then as follows,

Step 1. Given $f \in L^{2}\left(\Gamma_{\text {ext }}\right)$, choose an arbitrary initial guess

$$
\left(\varphi_{p}, t_{p}\right) \in L^{2}\left(\Gamma_{\text {int }}\right) \times L^{2}\left(\Gamma_{\text {int }}\right)
$$

Step 1.1. Solve the well-posed stochastic forward problem :

$$
\left\{\begin{array}{l}
\nabla \cdot\left(\sigma(x, \xi) \nabla v^{p}(x, \xi)\right)=0 \quad \text { in } D \times \Omega \\
\sigma(x, \xi) \frac{\partial v^{p}(x, \xi)}{\partial n}=0 \quad \text { on } \Gamma_{\text {ext }} \times \Omega \\
v^{p}(x, \xi)=t_{p} \quad \text { on } \Gamma_{\text {int }} \times \Omega
\end{array}\right.
$$

Step 1.2. Solve the stochastic adjoint problem:

$$
\left\{\begin{array}{l}
\nabla \cdot\left(\sigma(x, \xi) \nabla \lambda^{p}(x, \xi)\right)=0 \quad \text { in } D \times \Omega, \\
\lambda^{p}(x, \xi)=\sigma(x, \xi) \frac{\partial v(x, \xi)}{\partial n}-\varphi_{p} \quad \text { on } \Gamma_{\text {int }} \times \Omega, \\
\sigma(x, \xi) \frac{\partial \lambda^{p}(x, \xi)}{\partial n}=-(v(x, \xi)-f) \quad \text { on } \Gamma_{\mathrm{ext}} \times \Omega .
\end{array}\right.
$$

step 1.3. : We evaluate the gradient of the cost function:

$$
\nabla J\left(\varphi_{p}, t_{p}\right)=\left(E\left[\varphi^{p}-\sigma(x, \xi) \frac{\partial v^{p}(x, \xi)}{\partial n}\right], E\left[\sigma(x, \xi) \frac{\partial \lambda^{p}(x, \xi)}{\partial n}\right]\right)
$$


Step 1.4. Determine the descent direction $d_{p}$ as follows::

$$
\left\{\begin{array}{l}
\gamma_{p-1}=\frac{\left\|\nabla J\left(\varphi_{p}, t_{p}\right)\right\|^{2}}{\left\|\nabla J\left(\varphi_{p-1}, t_{p-1}\right)\right\|^{2}} \\
d_{p}:=\left(d_{1}^{p}, d_{2}^{p}\right)=-\nabla J\left(\varphi_{p}, t_{p}\right)+\gamma_{p-1} d_{p-1}
\end{array}\right.
$$

We compute: $\left(\varphi_{p+1}, t_{p+1}\right)=\left(\varphi_{p}, t_{p}\right)+\alpha_{p} d_{p}$

where the scalar $\alpha_{p}$ is obtained through a linear search by :

$$
\alpha_{p}=-\frac{E\left[\int_{\Gamma_{\mathrm{ext}}} z^{p}\left(v^{p}-f\right) d \Gamma_{\mathrm{ext}}\right]+E\left[\int_{\Gamma_{\mathrm{int}}}\left(\sigma \frac{\partial z^{p}}{\partial n}-d_{1}^{p}\right)\left(\sigma \frac{\partial v^{p}}{\partial n}-\varphi_{p}\right) d \Gamma_{\mathrm{int}}\right]}{E\left[\int_{\Gamma_{\mathrm{ext}}}\left(z^{p}\right)^{2} d \Gamma_{\mathrm{ext}}\right]+E\left[\int_{\Gamma_{\mathrm{int}}}\left(\sigma \frac{\partial z^{p}}{\partial n}-d_{1}^{p}\right)^{2} d \Gamma_{\mathrm{int}}\right]}
$$

where $z^{p}$ is the solution of the following stochastic problem:

$$
\left\{\begin{array}{l}
\nabla \cdot\left(\sigma(x, \xi) \nabla z^{p}(x, \xi)\right)=0 \quad \text { in } D \times \Omega \\
z^{p}(x, \xi)=d_{2}^{p} \text { on } \Gamma_{\text {int }} \times \Omega \\
\sigma(x, \xi) \frac{\partial z^{p}(x, \xi)}{\partial n}=0 \quad \text { on } \Gamma_{\mathrm{ext}} \times \Omega
\end{array}\right.
$$

Step 2. Having obtained $\left(\varphi_{p}, t_{p}\right)$ for $p \geq 0$, set $p=p+1$ and repeat from step 1.1 until the prescribed stopping criterion is satisfied. As a stopping criterion we choose $\left\|E\left[v^{p}(x, \xi)\right]-f\right\|_{L^{2}\left(\Gamma_{\text {ext }}\right)} \leq \epsilon$ or $\left\|\nabla J\left(\varphi_{p}, t_{p}\right)\right\| \leq \epsilon$. In practice, we take $\epsilon=0.001$.

\section{Results}

In this section we present the numerical results of the stochastic inverse problem. In order to assess the effect of the conductivity uncertainties of each of lungs and fat conductivities on the reconstructed electrical potential on the heart boundary, we start by generating our ground truth solution by solving the equation (3). For the sake of simplicity we take a harmonic function on the heart boundary: where the exact extracellular potential $u_{e x}(x, y)=\exp (x) \sin (y)$.

Since we assume that the uncertainty of the conductivity value follows a uniform probability density, as probability density functions $\left\{\Psi_{i}\right\}$ we use the Legendre polynomials defined on the interval $\Omega=[-1,1]$. We also suppose that the true conductivity uncertainty interval is centered by $\sigma_{T}$, the true conductivity. We propose to study three cases. In the first case we suppose that there is no uncertainties. In the second (respectively, third) case we suppose that all the conductivities values are known but the conductivity of fat (respectively, lungs) where we gradually increase the uncertainty from zero to $\pm 50 \%$ of the true conductivity value $\sigma_{T}$. We solve the stochastic inverse problem following the algorithm described in the previous section. We measure the effect of the uncertainties using relative error (RE) and the correlation coefficient (CC). In table1, we show the performance of the stochastic method. We used different level of uncertainties: $0 \%, \pm 3 \%, \pm 10 \%, \pm 20 \%, \pm 30 \%$ and $\pm 50 \%$. We find that the relative error of the inverse solution has been barely affected by the uncertainties of the fat conductivity even for high uncertainty levels. In fact the RE (respectively, CC ) is 0.1245 (respectively, 0.993) when there is no uncertainties and for $\pm 50 \%$ of uncertainties the $\mathrm{RE}$ (respectively, CC) is 0.1276 (respectively, 0.991). On the contrary the effect of the lung conductivity uncertainties is high: The RE increases from 0.1245 when we don't consider the uncertainties to 0.4852 when we have $\pm 50 \%$ of uncertainties on the lung conductivity. 


\begin{tabular}{|c|c|c|c|c|c|c|c|}
\hline & conductivity uncertainties & $0 \%$ & $\pm 3 \%$ & $\pm 10 \%$ & $\pm 20 \%$ & $\pm 30 \%$ & $\pm 50 \%$ \\
\hline relative error & fat & 0.1245 & 0.1245 & 0.1248 & 0.1248 & 0.1251 & 0.1276 \\
\hline & lungs & 0.1245 & 0.1263 & 0.1439 & 0.2208 & 0.3333 & 0.4852 \\
\hline Corr coeff & fat & 0.9930 & 0.9930 & 0.9945 & 0.9943 & 0.9980 & 0.991 \\
\hline & lungs & 0.9930 & 0.9933 & 0.9899 & 0.9767 & 0.9660 & 0.885 \\
\hline
\end{tabular}

Table 1: Relative error and correlation coefficient of the stochastic inverse solution for different levels of uncertainty on the fat and lungs conductivities .

The effect of the uncertainty on the correlation coefficient could also be qualitatively seen in Figure 2, where the pattern of the mean value of the stochastic inverse solution looks the same in Figure 2 (a) (no uncertainties) and (b) ( $\pm 50 \%$ of uncertainties on the fat conductivity) and different in Figure 2 (c) ( $\pm 50 \%$ of uncertainties on lungs conductivity). Similarly the effect of uncertainties on the relative error could be

(a)

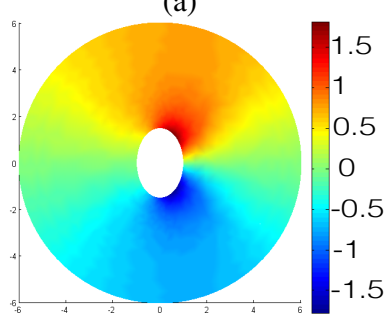

(b)

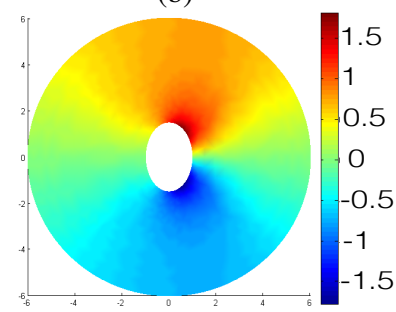

(c)

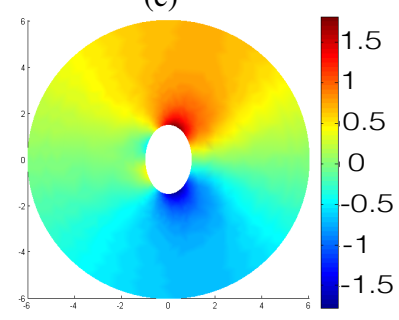

Fig. 2: Mean value of the SFEM inverse solution : No uncertainties(a), $\pm 50 \%$ uncertainty in the fat conductivity(b) and $\pm 50 \%$ uncertainty in lungs conductivity (c)

qualitatively seen in Figure 3. As shown in Table 1, the error does not change too much from no uncertainties (Figure 3 (a)) to $\pm 50 \%$ of fat conductivity uncertainty (Figure 3 (b)). Whereas the error is high for $\pm 50 \%$ of lungs conductivity uncertainty (Figure 3 (c)). The propagation of uncertainties from the conductivities to the the inverse problem solution is reflected in the deviation of the stochastic inverse solution from the ground truth presented in Figure 4. We remark that the error is concentrated in the heart boundary $\Gamma_{i n t}$, it reaches 0.8 for $\pm 50 \%$ of lungs conductivity uncertainty and 0.25 for $\pm 50 \%$ of fat conductivity uncertainty.

\section{Discussion}

Solving the inverse problem in electrocardiography imaging based on an optimal control problem allowed us to quantify the effect of the torso organs conductivity uncertainties using a stochastic finite element method. This work is the first mathematical study of the effect of the conductivity uncertainties on the inverse problem solution. Our results show that increasing the level of the fat conductivity uncertainty from zero to $\pm 50 \%$ of its original value does not alter the quality of the reconstructed potential. This is in line with the results presented in [13] for the forward problem when introducing $\pm 50 \%$ uncertainties in the fat conductivity. On the contrary, the results that we obtained for the uncertainties on the lungs conductivity show an important effect on the ECGI solution. In fact the relative error is about $50 \%$ when introducing $\pm 50 \%$ of uncertainty 
(a)

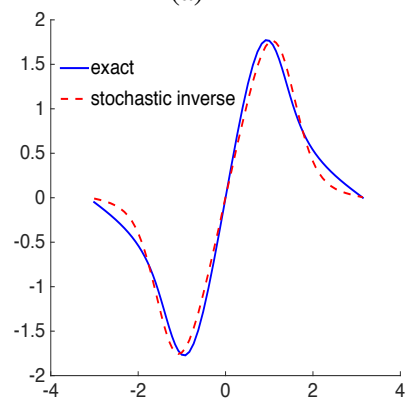

(b)

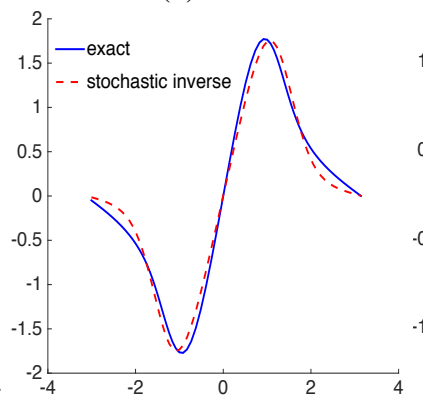

(c)

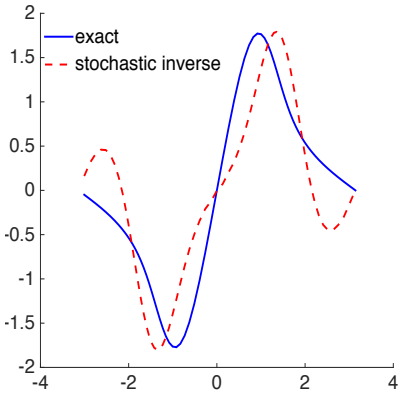

Fig. 3: Comparison of the SFEM solution to the ground truth on the heart boundary $\Gamma_{\text {int }}$ : (a) no uncertainties, (b) $\pm 50 \%$ uncertainties in the fat conductivity, (c) $\pm 50 \%$ uncertainties in lungs conductivity. Exact solution (blue continuous line). Stochastic inverse solution mean value (red dashed line). $\mathrm{X}$-axis polar coordinate angle from $-\pi$ to $\pi$. Y-axis value of the electrical potential on the boundary $\Gamma_{\text {int }}$.

(a)

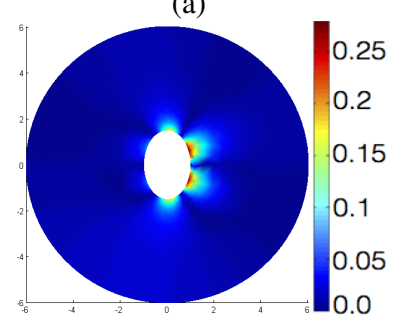

(b)

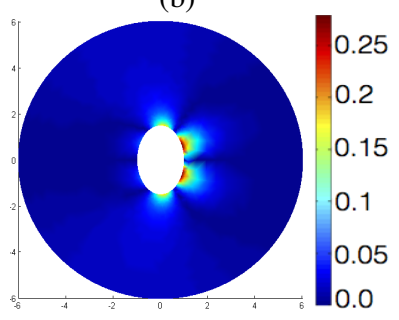

(c)

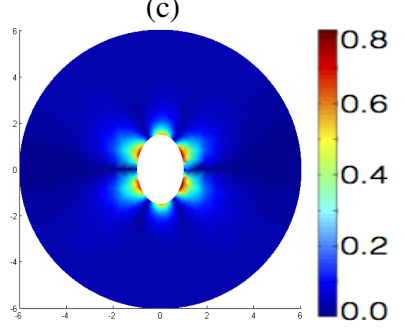

Fig. 4: Deviation of the SFEM inverse solution from the ground truth solution: No uncertainties (a), $\pm 50 \%$ uncertainty in the fat conductivity (b) and $\pm 50 \%$ uncertainty in lungs conductivity (c).

and the CC is significantly altered. This result is different from he results presented in [13] for the forward solution with $\pm 50 \%$ uncertainties in the lungs conductivity where the standard deviation does not exceed $3 \%$ of the mean value.

\section{5 conclusions}

In this work we presented a novel approach to study sensitivity to parameters values in data completion inverse problem and that could have application in a wide range of bioelectric and biomedical inverse problems resolution. We used a stochastic finite element method in order to take into account the variability of the conductivity values in the ECGI inverse problem formulated in an optimal control manner. We used a conjugate gradient method to solve this problem where the gradient of the cost function was computed using an adjoint method. We have described the different steps of the algorithm used to solve this stochastic inverse problem. The numerical simulation that we conducted in a simple 2D case showed that there is an important sensitivity of the solution to the lungs conductivity, whereas the uncertainties on the fat conductivity did not affect too much the inverse solution. It is still not clear if the difference in the sensitivity to the uncertainties is due to volume of the organs and/or their positions and proximity to the heart and/or the magnitude of their mean conductivities. This question would be a subject of a mathematical and numerical investigation as a continuation of this work. 


\section{Acknowledgments}

We would like to thank the LIRIMA Laboratory which financially supported the teams ANO and EPICARD to perform this work.

\section{References}

1. Shah, A.J., Lim, H.S., Yamashita, S., Zellerhoff, S., Berte, B., Mahida, S., Hooks, D., Aljefairi, N., Derval, N., Denis, A., et al.: Non invasive ecg mapping to guide catheter ablation. JAFIB: Journal of Atrial Fibrillation 7(3) (2014)

2. Hadamard, J.: Lectures on Cauchy's problem in linear partial differential equations. Yale University Press, New Haven (1923)

3. Ghosh, S., Rudy, Y.: Application of 11-norm regularization to epicardial potential solution of the inverse electrocardiography problem. Annals of Biomedical Engineering 37(5) (2009) 902912

4. Zakharov, E., Kalinin, A.: Algorithms and numerical analysis of dc fields in a piecewisehomogeneous medium by the boundary integral equation method. Computational Mathematics and Modeling 20(3) (2009) 247-257

5. Li, G., He, B.: Localization of the site of origin of cardiac activation by means of a heartmodel-based electrocardiographic imaging approach. Biomedical Engineering, IEEE Transactions on 48(6) (2001) 660-669

6. Doessel, O., Jiang, Y., Schulze, W.H.: Localization of the origin of premature beats using an integral method. International Journal of Bioelectromagnetism 13 (2011) 178-183

7. Zemzemi, N., Dubois, R., Coudiere, Y., Bernus, O., Haissaguerre, M.: A machine learning regularization of the inverse problem in electrocardiography imaging. In: Computing in Cardiology Conference (CinC), 2013. (Sept 2013) 1135-1138

8. Hansen, P.C., O'Leary, D.P.: The use of the l-curve in the regularization of discrete ill-posed problems. SIAM Journal on Scientific Computing 14(6) (1993) 1487-1503

9. Foster, K.R., Schwan, H.P.: Dielectric properties of tissues and biological materials: a critical review. Critical reviews in biomedical engineering 17(1) (1988) 25-104

10. Faes, T., Van Der Meij, H., De Munck, J., Heethaar, R.: The electric resistivity of human tissues (100 hz-10 mhz): a meta-analysis of review studies. Physiological measurement 20(4) (1999) R1

11. Gabriel, S., Lau, R., Gabriel, C.: The dielectric properties of biological tissues: Ii. measurements in the frequency range $10 \mathrm{hz}$ to $20 \mathrm{ghz}$. Physics in medicine and biology 41(11) (1996) 2251

12. Van Oosterom, A., Huiskamp, G.: The effect of torso inhomogeneities on body surface potentials quantified using tailored geometry. Journal of electrocardiology 22(1) (1989) $53-$ 72

13. Geneser, S.E., Kirby, R.M., MacLeod, R.S.: Application of stochastic finite element methods to study the sensitivity of ecg forward modeling to organ conductivity. Biomedical Engineering, IEEE Transactions on 55(1) (2008) 31-40

14. Weber, F.M., Keller, D.U., Bauer, S., Seemann, G., Lorenz, C., Dossel, O.: Predicting tissue conductivity influences on body surface potentialsan efficient approach based on principal component analysis. Biomedical Engineering, IEEE Transactions on 58(2) (2011) 265-273

15. Aboulä̈ch, R., Abda, A.B., Kallel, M., Bal, G., Jollivet, A., Bresson, X., Chan, T.F., Flenner, A., Hewer, G.A., Kenney, C.S., et al.: Missing boundary data reconstruction via an approximate optimal control. Inverse Problems and Imaging 2(4) (2008)

16. Le Maître, O.P., Reagan, M.T., Najm, H.N., Ghanem, R.G., Knio, O.M.: A stochastic projection method for fluid flow: Ii. random process. Journal of computational Physics 181(1) (2002) 9-44

17. Andrieux, S., Baranger, T., Abda, A.B.: Solving cauchy problems by minimizing an energylike functional. Inverse problems 22(1) (2006) 115 